\title{
Impurity-induced orbital magnetization in a Rashba electron gas
}

\author{
Juba Bouaziz, ${ }^{*}$ Manuel dos Santos Dias, Filipe Souza Mendes Guimarães, Stefan Blügel, and Samir Lounis \\ Peter Grünberg Institut and Institute for Advanced Simulation, Forschungszentrum Jülich and JARA, \\ 52425 Jülich, Germany
}

(Received 6 June 2018; revised manuscript received 28 August 2018; published 24 September 2018)

\begin{abstract}
We investigate the induced orbital magnetization density in a Rashba electron gas with magnetic impurities. Relying on classical electrodynamics, we obtain this quantity through the bound currents composed of paramagnetic and diamagneticlike contributions which emerge from the spin-orbit interaction. Similar to Friedel charge ripples, the bound currents and the orbital magnetization density oscillate as a function of distance away from the impurity with characteristic wavelengths defined by the Fermi energy and the strength of the Rashba spin-orbit interaction. The net induced orbital magnetization was found to be of the order of magnitude of its spin counterpart. In addition to the exploration of the impact of the electronic filling of the impurity states, we investigate and analyze the orbital magnetization induced by an equilateral frustrated trimer in various noncollinear magnetic states. On the one hand, we confirm that nonvanishing three-spin chiralities generate a finite orbital magnetization density. On the other hand, higher-order contributions lead to multiple-spin chiralities affecting nontrivially and significantly the overall magnitude and sign of the orbital magnetization. This study substantiates the notion that the orbital degrees of freedom are an essential aspect of nanoscale magnetism, calling for further theoretical and experimental attention.
\end{abstract}

DOI: 10.1103/PhysRevB.98.125420

\section{INTRODUCTION}

The inversion symmetry breaking at surfaces and interfaces leads to the emergence of a wide variety of phenomena. Its signature can be detected experimentally through different physical quantities [1-3]. In combination with the spin-orbit (SO) interaction, it leads to the Rashba effect, which consists of an energy and spin splitting of the surface and interface states [3-5]. The Rashba effect was observed experimentally at noble-metallic surfaces using angle-resolved photoemission spectroscopy [6-8] through the energy dispersion imaging of the Rashba spin splitting. Since its first observations, several studies were devoted to this effect, as reported in several reviews [3,9]. For instance, it was shown that the Rashba spin splitting can be manipulated by material engineering [10-12]. In the case of a $\mathrm{Bi}$ monolayer deposited on $\mathrm{Si}(111)$, the splitting can be very large [10].

An alternative way to probe the spin splitting of the surface states is using the Friedel oscillations [13] resulting from the scattering of the surface electrons off defects or impurities. They can be accessed experimentally via scanning tunneling microscopy (STM) [14,15]. Although it was shown theoretically that no signature of the Rashba spin splitting can be observed in the charge density surrounding a single nonmagnetic impurity [16], larger ensembles of impurities such as quantum corrals do enable its detection [17]. Lounis et al. [18] showed that the introduction of a magnetic impurity into a Rashba electron gas causes Friedel oscillations in the spin magnetization density where a signature of the spin splitting can be observed: The induced spin magnetization

\footnotetext{
*j.bouaziz@fz-juelich.de
}

exhibits a skyrmionlike spin texture. In addition, the magnetic impurities also generate finite ground-state spin currents, leading to the emergence of the chiral Dzyaloshinskii-Moriya interaction [19-21]. The latter is the key ingredient for the stabilization of topological spin textures such as magnetic skyrmions [22-25].

In addition to the induced spin magnetization and spin currents, the combination of the Rashba SO interaction and breaking of time-reversal symmetry (due to magnetic impurities or magnetic fields) generates bound charge currents and also a large orbital magnetic susceptibility [26,27]. These bound currents were explored in the context of magnetic impurities and ferromagnetic islands coupled to superconductors [28], and as they represent electrons moving in a closed circuit, they should also lead to a finite orbital magnetization. In classical electrodynamics, the bound currents and orbital magnetization are related via [29]

$$
\vec{j}(\vec{r})=\vec{\nabla}_{\vec{r}} \times \vec{m}_{l}(\vec{r})
$$

where $\vec{m}_{l}(\vec{r})$ is the orbital magnetization density and $\vec{j}(\vec{r})$ is the bound current density. The spin contribution to the magnetization is additive to the orbital one and was investigated in Ref. [18]. In equilibrium, $\vec{j}(\vec{r})$ is nondissipative and fulfills the continuity equation for the electron density $\rho(\vec{r})$ [see Eq. (5)]. Recently, it was shown that a finite orbital magnetization emerges for certain types of magnetic structures even in the absence of the spin-orbit interaction [30-32]. It then arises from the scalar spin chirality of the magnetic texture, $\mathcal{C}_{i j k}=\vec{S}_{i} \cdot\left(\vec{S}_{j} \times \vec{S}_{k}\right)$, with $\vec{S}_{i}$ being the spin magnetic moment direction at site $i$. Indeed, noncollinear spin textures can be viewed as an emergent magnetic field that couples the spin and orbital degrees of freedom [33,34], mimicking the effects of the SO interaction. Moreover, for large magnetic skyrmions, 
the chirality-driven orbital magnetization is quantized and becomes topological (i.e., not affected by continuous deformations of the magnetic texture) and might be accessed experimentally via x-ray magnetic circular dichroism (XMCD) [31]. The chiral orbital magnetization was also found in periodic systems [35-37] as well as in continuous topological structures deposited on a Rashba electron gas [38]. Remarkably, the time-reversal-invariant Rashba electron gas can also be seen as having a compensated orbital magnetization [39].

In this paper, we investigate the induced orbital magnetization generated when magnetic impurities are deposited on a Rashba electron gas. This model was well characterized in Ref. [40], which studied the long-range interactions between the magnetic impurities mediated by the spin degrees of freedom of the Rashba electron gas. Here, we turn our attention to their orbital degrees of freedom and study how they lead to orbital magnetism. We consider a single impurity or a trimer with a noncollinear spin state and show that the orbital and spin magnetizations can be of comparable magnitude. Furthermore, we demonstrate that higher-order spin chiralities can provide a substantial contribution to the induced orbital magnetization generated by clusters involving more than one impurity. This study substantiates the notion that the orbital degrees of freedom are an essential contribution to nanoscale magnetism and merit further theoretical and experimental attention. The paper is structured as follows: First, we discuss the bound currents and their different contributions (paramagnetic and diamagnetic), which are evaluated analytically for the single-impurity case. Second, the induced orbital magnetization density is computed starting from the bound currents by numerically solving a Poisson equation. The impact of the impurity's nature on the orbital magnetization from tuning the scattering phase shifts is also considered. Finally, we compute the orbital magnetization for a magnetic trimer in an equilateral triangle with and without the SO interaction and provide functional forms connecting the spin impurity moments to the orbital magnetization.

\section{RASHBA MODEL}

The SO interaction leads, in a structure-asymmetric environment such as surfaces and interfaces, to a spin splitting of the degenerate eigenstates for the two-dimensional free-electron gas. The model of Bychkov and Rashba [4,5] describes this splitting by adding a linear term in momentum $\vec{p}$ to the kinetic energy of the free electrons. The so-called Rashba Hamiltonian is given by

$$
\boldsymbol{H}_{\mathrm{R}}=\frac{p_{x}^{2}+p_{y}^{2}}{2 m^{*}} \mathbb{1}_{2}-\frac{\alpha_{\mathrm{R}}}{\hbar}\left(\boldsymbol{\sigma}_{x} p_{y}-\boldsymbol{\sigma}_{y} p_{x}\right),
$$

where $\left\{p_{x}, p_{y}\right\}$ are the components of the momentum operator $\vec{p}$ in Cartesian coordinates of the surface plane whose normal points along $\vec{e}_{z}$ and $m^{*}$ is the effective mass of the electron. $\sigma_{x}$ and $\sigma_{y}$ are Pauli matrices, and $\mathbb{1}_{2}$ is the unit matrix in spin space with a global spin frame of reference parallel to the $z$ axis. $\alpha_{\mathrm{R}}$ is known as the Rashba parameter and represents the strength of the SO interaction. The linear term in Eq. (2) is induced by a SO gauge field given by $[41,42]$

$$
\overrightarrow{\boldsymbol{A}}_{\mathrm{R}}=\frac{m^{*} \alpha_{\mathrm{R}}}{e \hbar}\left(-\sigma_{y}, \sigma_{x}\right) \text {, }
$$

where $e$ is the electron charge. Using this SO gauge field, the Hamiltonian is expressed as

$$
\boldsymbol{H}_{\mathrm{R}}=\frac{\left(\vec{p}-e \overrightarrow{\boldsymbol{A}}_{\mathrm{R}}\right)^{2}}{2 m^{*}}-V_{\mathrm{R}},
$$

with $V_{\mathrm{R}}=\frac{m^{*} \alpha_{\mathrm{R}}^{2}}{\hbar^{2}}$ being a constant. Since $\left[\boldsymbol{A}_{\mathrm{R}}^{x}, \boldsymbol{A}_{\mathrm{R}}^{y}\right] \neq 0, \overrightarrow{\boldsymbol{A}}_{\mathrm{R}}$ is a non-Abelian gauge field, which complicates any possible approach relying on gauge transformations [42]. Starting from the time-dependent Schrödinger equation, $i \hbar \frac{\partial \psi(\vec{r}, t)}{\partial t}=$ $\boldsymbol{H}_{\mathrm{R}} \psi(\vec{r}, t)$, we arrive at a continuity equation relating the electron charge density $\rho(\vec{r}, t)=|\psi(\vec{r}, t)|^{2}$ and the current density $\vec{j}(\vec{r}, t)$,

$$
\frac{\partial \rho(\vec{r}, t)}{\partial t}+\vec{\nabla}_{\vec{r}} \cdot \vec{j}(\vec{r}, t)=0 .
$$

The current density is the expectation value of the current operator [43],

$$
\overrightarrow{\boldsymbol{j}}=\lim _{\vec{r}^{\prime} \rightarrow \vec{r}}\left(\frac{\hbar\left(\vec{\nabla}_{\vec{r}}-\vec{\nabla}_{\vec{r}^{\prime}}\right)}{2 m^{*} i} \mathbb{1}_{2}-\frac{e}{m^{*}} \overrightarrow{\boldsymbol{A}}_{\mathrm{R}}\right),
$$

written in a form suitable to operate on the off-diagonal elements of the density matrix,

$$
\begin{aligned}
\vec{j}(\vec{r}, t) & =\langle\vec{j}\rangle=\operatorname{Tr} \vec{j} \psi(\vec{r}, t) \psi^{\dagger}\left(\vec{r}^{\prime}, t\right) \\
& =-\frac{1}{\pi} \operatorname{Im} \operatorname{Tr} \vec{j} \boldsymbol{G}\left(\vec{r}, \vec{r}^{\prime} ; t, t^{+}\right),
\end{aligned}
$$

where $\boldsymbol{G}\left(\vec{r}, \vec{r}^{\prime} ; t, t^{+}\right)$is the retarded single-particle Green's function, which will be used in the following. The first term in Eq. (6) is the paramagnetic contribution to the current operator, while the second term is a diamagneticlike contribution arising from the SO gauge field. Both parts are included in the calculations presented in this paper. Furthermore, when starting from the Dirac Hamiltonian and performing an expansion in the nonrelativistic limit, one finds an extra contribution to the current operator coming from the Zeeman term of the Hamiltonian, $\overrightarrow{\boldsymbol{j}}_{\text {Zeeman }}=\frac{\hbar}{2 m^{*}} \lim _{\vec{r}^{\prime} \rightarrow \vec{r}} \vec{\nabla} \vec{r} \times \overrightarrow{\boldsymbol{\sigma}}$. It may be induced either by a magnetic field or a finite magnetization [44]. However, this term is not included in our discussion since it does not contribute to the orbital magnetization, which is the quantity of interest in this paper.

\section{BOUND CURRENTS EMERGING FROM A SINGLE MAGNETIC IMPURITY ON A RASHBA ELECTRON GAS}

The introduction of magnetic impurities into the system leads to the breaking of time-reversal symmetry, which in the presence of SO interaction is expected to induce a finite orbital magnetization [29]. The magnetic impurities are embedded into the Rashba electron gas using a Green's function approach in real space via the Dyson equation

$$
\begin{aligned}
\boldsymbol{G}\left(\vec{r}, \vec{r}^{\prime}, \varepsilon\right)= & \boldsymbol{G}^{\mathrm{R}}\left(\vec{r}, \vec{r}^{\prime}, \varepsilon\right) \\
& +\sum_{i j} \boldsymbol{G}^{\mathrm{R}}\left(\vec{r}, \vec{r}_{i}, \varepsilon\right) \boldsymbol{\tau}_{i j}(\varepsilon) \boldsymbol{G}^{\mathrm{R}}\left(\vec{r}_{j}, \vec{r}^{\prime}, \varepsilon\right),
\end{aligned}
$$

relating the Green's function of the Rashba electron gas $\boldsymbol{G}^{\mathrm{R}}\left(\vec{r}, \vec{r}^{\prime}, \varepsilon\right)$ (see Appendix A for an explicit expression) to the Green's function of the Rashba electron gas with impurities $\boldsymbol{G}\left(\vec{r}, \vec{r}^{\prime}, \varepsilon\right)$ through the scattering path operators $\boldsymbol{\tau}_{i j}(\varepsilon)(i, j$ 
running over the impurities). The latter describes single- and multiple-scattering processes experienced by the electrons at the impurities and can be computed from the transition matrices for isolated impurities ( $t$ matrix) $\boldsymbol{t}_{i}(\varepsilon)$ as

$$
\begin{aligned}
\boldsymbol{\tau}_{i j}(\varepsilon)= & \boldsymbol{t}_{i}(\varepsilon) \delta_{i j} \\
& +\sum_{k} \boldsymbol{t}_{i}(\varepsilon) \boldsymbol{G}_{i k}^{\mathrm{R}}(\varepsilon)\left(1-\delta_{i k}\right) \boldsymbol{\tau}_{k j}(\varepsilon) .
\end{aligned}
$$

Furthermore, considering that the Fermi wavelength of the Rashba electrons is much larger than the spatial extension of the impurities, we employ the $s$-wave approximation [15]. When the magnetic moment of the impurity is along the $z$ axis, the transition matrix is fully characterized by two quantities, $t_{i}^{\sigma}(\varepsilon)=\frac{i \hbar}{m^{*}}\left(e^{2 i \delta_{i}^{\sigma}(\varepsilon)}-1\right) . \sigma=\uparrow, \downarrow$ are labels for the spin projection of an electron belonging to the surface along the quantization axis defined by the orientation of the magnetic moment of the impurity, and $\delta_{i}^{\sigma}(\varepsilon)$ is the corresponding scattering phase shift. For a general orientation, the transition matrix becomes

$$
\boldsymbol{t}_{i}(\varepsilon)=\boldsymbol{U}_{i}\left(\begin{array}{cc}
t_{i}^{\uparrow}(\varepsilon) & 0 \\
0 & t_{i}^{\downarrow}(\varepsilon)
\end{array}\right) \boldsymbol{U}_{i}^{\dagger},
$$

with $\boldsymbol{U}_{i}$ being the spin rotation matrix transforming the spin quantization axis from the $z$ axis to the orientation of the magnetic moment of the impurity. This form can be derived by assuming a contact interaction between the electrons and the impurity, including magnetic and nonmagnetic scattering. The phase shifts encode the chemical and magnetic nature of the impurity. For instance, Fe can be approximated by $\delta_{i}^{\uparrow}=\pi$ and $\delta_{i}^{\downarrow}=\frac{\pi}{2}$ [40].

When only one impurity is present, Eq. (9) reduces to $\boldsymbol{\tau}_{i j}(\varepsilon)=\boldsymbol{t}_{i}(\varepsilon) \delta_{i j}$, and $\vec{j}(\vec{r})$ can be computed analytically. Placing the impurity at the origin with its magnetic moment pointing perpendicular to the surface (i.e., along the $z$ axis), the cylindrical symmetry of the Rashba electron gas is preserved, and the current density in cylindrical coordinates $\vec{r}=$ $(r \cos \theta, r \sin \theta)$ reads

$$
\begin{aligned}
\vec{j}(\vec{r})= & -\frac{\hbar}{m^{*} \pi} \operatorname{Im} \int_{0}^{\varepsilon_{\mathrm{F}}} d \varepsilon\left[\frac{G_{\mathrm{ND}}^{2}(r, \varepsilon)}{r}\right. \\
& \left.-\frac{2 m^{*} \alpha_{\mathrm{R}}}{\hbar^{2}} G_{\mathrm{D}}(r, \varepsilon) G_{\mathrm{ND}}(r, \varepsilon)\right] \Delta t_{i}(\varepsilon) \vec{e}_{\theta} .
\end{aligned}
$$

Here, $\vec{e}_{\theta}=(\sin \theta,-\cos \theta)$ is the azimuthal unit vector at point $\vec{r}$. $G_{\mathrm{D}}(r, \varepsilon)$ and $G_{\mathrm{ND}}(r, \varepsilon)$ are functions of the distance $r=|\vec{r}|$ and energy $\varepsilon$ and represent the diagonal and offdiagonal parts of the Rashba Green's function in spin space, respectively. More details on this derivation are given in Appendix $\mathrm{A}$. The dependence of the current on the intrinsic properties of the impurity is entirely encoded in $\Delta t_{i}(\varepsilon)=t_{i}^{\uparrow}(\varepsilon)-$ $t_{i}^{\downarrow}(\varepsilon)$. This result reveals, in a clear fashion, that a finite orbital magnetization requires a spin magnetization/magnetic field breaking time-reversal symmetry [i.e., $t_{i}^{\uparrow}(\varepsilon) \neq t_{i}^{\downarrow}(\varepsilon)$ ] and a broken space-inversion symmetry environment with the SO interaction [i.e., $G_{\mathrm{ND}}(r, \varepsilon) \neq 0$ ]. The first term in Eq. (11) represents the paramagnetic part of the current density, while the second is the diamagnetic one. $\vec{j}(\vec{r})$ has no radial component, thus swirling around the magnetic impurity. Similar results were obtained for magnetic impurities deposited on superconductors with Rashba spin-orbit interaction [28].

A comment concerning the energy integration in Eq. (11) is in order. The integration is performed only from $\left[0, \varepsilon_{\mathrm{F}}\right]$. The energy range $\left[-\varepsilon_{\mathrm{R}}, 0\right]$, with $\varepsilon_{\mathrm{R}}=\frac{m^{*} \alpha_{\mathrm{R}}^{2}}{2 \hbar^{2}}$ being the Rashba energy, is not included for two reasons. First, for realistic values of the Rashba parameter we have $\varepsilon_{\mathrm{R}} \ll \varepsilon_{\mathrm{F}}$, so this energy range is very small. Second, although this energy range contains a Van Hove singularity, a careful analysis shows that the $t$ matrix cancels the singularity and leads to a smooth energy dependence of the Green's function [40,45]. Combining both arguments, we conclude that one can safely neglect the contribution from this energy range.

In Fig. 1(a), we show the ground-state charge currents induced by a single Fe impurity deposited on the Rashba surface states of a $\mathrm{Au}(111)$ surface, computed from Eq. (11). The Rashba model parameters are $\alpha_{\mathrm{R}}=-0.4 \mathrm{eV} \AA, m^{*}=$ $0.26 m_{\mathrm{e}}\left(m_{\mathrm{e}}\right.$ is the electron mass), and $\varepsilon_{\mathrm{F}}=410 \mathrm{meV}$ [17]. These swirling bound currents are dissipationless (i.e., with zero divergence) with an oscillating amplitude reminiscent of the Friedel oscillations present in the charge and spin densities. A cut at $y=0$ is shown in Fig. 1(b), where the oscillating current density displays a beating effect at $x \sim$ $60 \AA$ similar to the one observed in the spin magnetization density and magnetic exchange interactions characterizing single impurities embedded in a Rashba electron gas $[18,40]$. Two wavelengths are at play in settling the oscillatory behavior of the current density: a short one given by the Fermi wavelength $\lambda_{\mathrm{F}} \sim 18.5 \AA$ and a long one induced by the SO interaction $\lambda_{\mathrm{R}} \sim 130 \AA$. This behavior can be understood when considering the analytical form of the current density obtained in the asymptotic limit [i.e., expanding $G_{\mathrm{D}}(r, \varepsilon)$ and $G_{\mathrm{ND}}(r, \varepsilon)$ for $\left.r \rightarrow \infty\right]$ :

$$
\begin{aligned}
\vec{j}(r)= & -\frac{m^{*}}{\hbar^{3} \pi r} \operatorname{Im} \Delta t_{i}\left\{6 k_{\mathrm{R}}^{2}\left[\mathrm{CI}\left(2 k_{\mathrm{F}} r\right)-\operatorname{CI}\left(2\left|k_{\mathrm{R}}\right| r\right)\right]\right. \\
& \left.-2 k_{\mathrm{R}}^{2}\left[\frac{\sin \left(2 k_{\mathrm{F}} r\right)}{2 k_{\mathrm{F}} r}-\frac{\sin \left(2\left|k_{\mathrm{R}}\right| r\right)}{2\left|k_{\mathrm{R}}\right| r}\right]\right\} \vec{e}_{\theta} \\
& +\frac{2\left(m^{*}\right)^{2} \alpha_{\mathrm{R}}}{\hbar^{4} \pi} \operatorname{Im} \Delta t_{i}\left\{4 k_{\mathrm{R}}^{2}\left[\operatorname{SI}\left(2 k_{\mathrm{F}} r\right)-\mathrm{SI}\left(2\left|k_{\mathrm{R}}\right| r\right)\right]\right. \\
& \left.+\frac{1}{r^{2}}\left[\sin \left(2 k_{\mathrm{F}} r\right)-\sin \left(2\left|k_{\mathrm{R}}\right| r\right)\right]\right\} \vec{e}_{\theta} .
\end{aligned}
$$

$\mathrm{CI}(x)$ and $\mathrm{SI}(x)$ represent the sine and cosine integrated functions of $x$, while $k_{\mathrm{R}}=\frac{m^{*} \alpha_{\mathrm{R}}}{\hbar^{2}}$. Equation (12) shows that in the asymptotic limit, the current density oscillates with two different wavelengths, $\lambda_{\mathrm{F}}=\frac{\pi}{k_{\mathrm{F}}}$ and $\lambda_{\mathrm{R}} \propto \frac{\pi}{k_{\mathrm{R}}}$.

\section{METHOD FOR THE EVALUATION OF THE ORBITAL MAGNETIZATION}

In the previous section, we showed that a system with broken time- and space-inversion symmetry hosts groundstate charge currents. These currents give rise to a finite orbital magnetization within the Rashba electron gas. In the absence of free charge currents and time-dependent external fields, the orbital magnetization density $\vec{m}_{l}(\vec{r})$ is related to the ground-state charge current via Eq. (1). Let us begin 

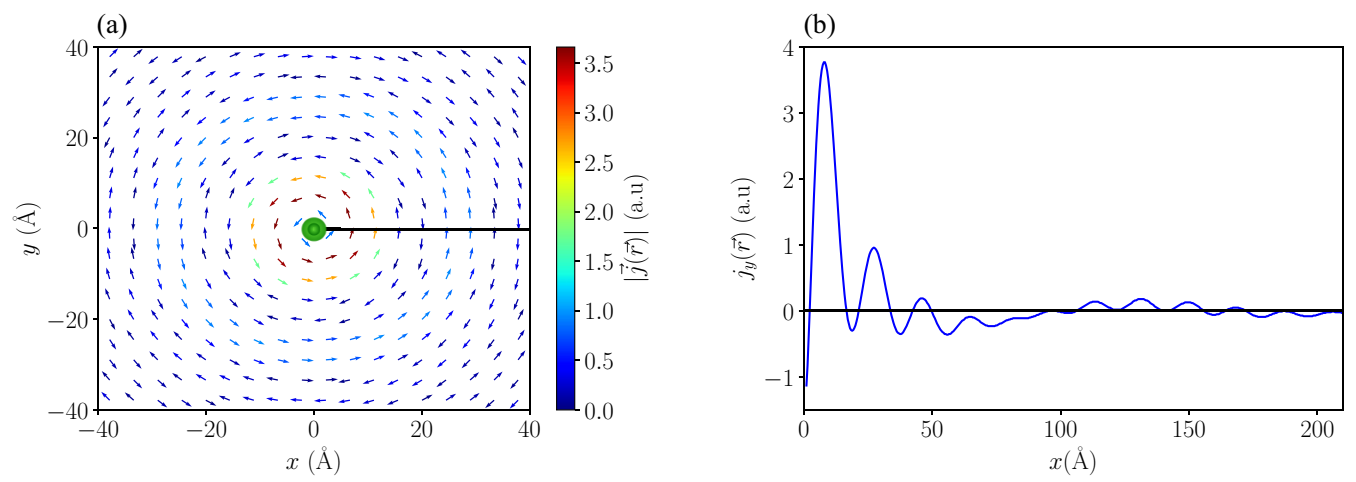

FIG. 1. Ground-state charge currents induced by a single Fe impurity on $\mathrm{Au}(111)$ with a magnetic moment perpendicular to the surface plane (along the $z$ axis). $\alpha_{\mathrm{R}}=-0.4 \mathrm{eV} \AA, m^{*}=0.26 m_{\mathrm{e}}$, and $\varepsilon_{\mathrm{F}}=410 \mathrm{meV}$ [17] are the Rashba model parameters for the Au(111) surface state. The Fe impurity is considered in the $s$-wave approximation [40]. (a) The dissipationless currents are swirling around the magnetic impurity, in agreement with the continuity equation and the axial symmetry of the system. (b) Evolution of the $y$ component of the current density as a function of the distance from the impurity. It displays an oscillatory behavior with two wavelengths, $\lambda_{\mathrm{F}} \sim 18.5 \AA$ and $\lambda_{\mathrm{R}} \sim 130 \AA$.

by showing that there is no indeterminacy in this relation, contrary to the three-dimensional case [46]. Due to the twodimensional geometry, the current density lies in the $x y$ plane, so the orbital magnetization is restricted to the $z$ direction. The standard indeterminacy in Eq. (1) consists of adding the gradient of an arbitrary function to the orbital magnetization, which does not affect the current density. Since only the $z$ component of the gradient is compatible with this geometry and it vanishes identically, there is no remaining freedom in the definition of the orbital magnetization density. Therefore, we can use Eq. (1) to define the orbital magnetization density by rewriting it as a Poisson equation [recall that $\vec{r}=(x, y)$ ],

$$
\begin{aligned}
\vec{\nabla} \times \vec{j}(\vec{r}) & =\vec{\nabla} \times \vec{\nabla} \times \vec{m}_{l}(\vec{r}), \\
& =\vec{\nabla}\left(\vec{\nabla} \cdot \vec{m}_{l}(\vec{r})\right)-\nabla^{2} \vec{m}_{l}(\vec{r}) .
\end{aligned}
$$

In this two-dimensional geometry, we finally obtain

$$
\partial_{x} j_{y}(\vec{r})-\partial_{y} j_{x}(\vec{r})=-\nabla^{2} m_{l, z}(\vec{r}),
$$

which can be solved numerically using a Fourier series in a large finite-simulation box. The Fourier components $m_{l, z}(\vec{k})$ of the orbital magnetization are

$$
m_{l, z}(\vec{k})=i \frac{k_{y} j_{x}(\vec{k})-k_{x} j_{y}(\vec{k})}{k_{x}^{2}+k_{y}^{2}},
$$

where $\vec{k}=\left(k_{x}, k_{y}\right)$ and $j_{\alpha}(\vec{k})$ is the Fourier transform of $j_{\alpha}(\vec{r})$, defined as

$$
j_{\alpha}(\vec{k})=\sum_{i=1}^{N_{\mathrm{r}}} j_{\alpha}\left(\vec{r}_{i}\right) e^{\vec{k} \cdot \vec{r}_{i}} .
$$

In practice, we consider $\mathrm{Fe}$ impurities deposited on $\mathrm{Au}(111)$ [40] in a box of $210 \times 210 \AA^{2}$ divided into a grid of $N_{\mathrm{r}}=1000 \times 1000$ points in real space. In our calculations, the Fermi wavelength is set to $\lambda_{\mathrm{F}} \sim 18.5 \AA$. The ratio between $\lambda_{\mathrm{F}}$ and the grid spacing is thus 0.01 , which was found to lead to converged results. This large box also ensures that $\vec{j}(\vec{r}) \simeq 0$ at the edges of the box to avoid interactions between the impurity and its periodic copies. Last, $m_{l, z}(\vec{k})$ is Fourier transformed back to real space, providing $m_{l, z}(\vec{r})$.

\section{ORBITAL MAGNETIZATION INDUCED BY A SINGLE IMPURITY}

We now discuss the orbital magnetization obtained for the setup discussed in Sec. IV in the presence of a single magnetic impurity with a spin moment pointing perpendicular to the plane ( $z$ axis) and also when it points in the plane along the $x$ axis. The orbital magnetization densities obtained using Eq. (14) are shown in Figs. 2(a) and 2(b). In the first case, we observe isotropic Friedel oscillations in the induced orbital magnetization around the $\mathrm{Fe}$ impurity since the spin moment does not break the cylindrical symmetry. Similar to the current density, $m_{l, z}(\vec{r})$ oscillates with two characteristic wavelengths, $\lambda_{\mathrm{F}}$ and $\lambda_{\mathrm{R}}$. These oscillations decay as $\frac{1}{r}$, which is slower than the induced spin magnetization [18]. Nonetheless, $m_{l, z}(\vec{r})$ is one order of magnitude smaller than the induced spin magnetization density. The net orbital and spin magnetizations are $M_{l, z}=-0.58 \mu_{\mathrm{B}}$ and $M_{s, z}=2.11 \mu_{\mathrm{B}}$, respectively.

For the in-plane orientation depicted in Fig. 2(b), where the cylindrical symmetry is broken, two oscillation wavelengths are also found: $\lambda_{\mathrm{F}}$ and $\lambda_{\mathrm{R}}$. The orbital magnetization density $m_{l, z}(\vec{r})$, however, oscillates around a positive (negative) value for $x<0(x>0)$. The oscillations are less pronounced than in the case where the impurity has a moment along the $z$ axis in Fig. 2(a). Nevertheless, the order of magnitude and the asymptotic decay of $m_{l, z}(\vec{r})$ at large distances are similar in both cases. Furthermore, when the spin moment points in the plane, $m_{l, z}(x, y)=-m_{l, z}(-x, y)$, and therefore, the total induced orbital magnetization sums to zero. The net induced spin magnetization vanishes as well.

\section{ORBITAL MAGNETIZATION FOR DIFFERENT TYPES OF IMPURITIES}

Here, we consider a single impurity with $\vec{S}_{i} \| z$ axis and investigate the dependence of the induced orbital magnetization on the nature of the magnetic impurities. This is achieved by shifting the position of the impurity resonance with respect to the Fermi energy of the Rashba electron gas, which represents different charge and spin states of the impurity. The impurities are modeled using a scattering phase shift $\delta_{i}^{\sigma}(\varepsilon)$ that can be 
(a)

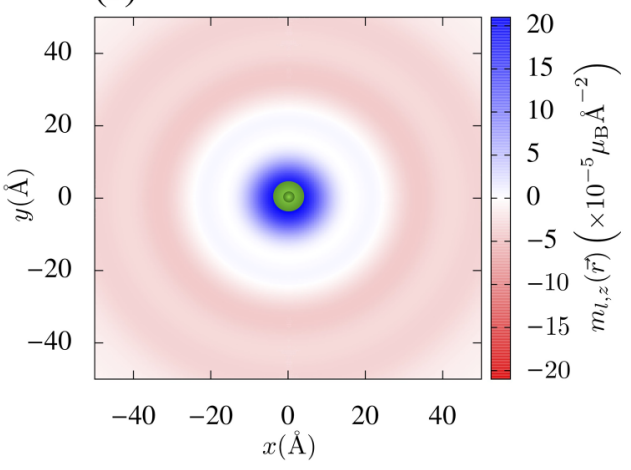

(b)

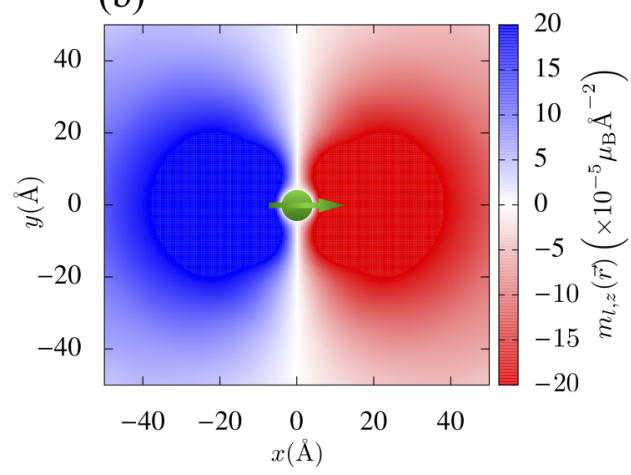

FIG. 2. Induced orbital magnetization map for a single Fe adatom deposited on a Rashba electron gas using the same model parameters as in Sec. III. The Fe impurity taken in the $s$-wave approximation [15] is represented by a green sphere located at the origin, while its magnetic moment is represented by a green arrow. (a) When the impurity spin moment points along the $z$ direction, the induced orbital magnetization consists of concentric rings centered around the Fe impurity oscillating with two characteristic wavelengths $\lambda_{\mathrm{F}}$ and $\lambda_{\mathrm{R}}$. (b) When the impurity spin moment lies in the plane, along the $x$ direction, the orbital magnetization is strongly anisotropic since $m_{l}^{z}$ is positive (negative) for negative (positive) $x$.

related to its local density of states $n_{i}(\varepsilon)$ via the Friedel sum rule [13]

$$
n_{i}(\varepsilon)=\frac{1}{\pi} \frac{d \delta_{i}^{\sigma}(\varepsilon)}{d \varepsilon} .
$$

We focus on $3 d$ transition-metal impurities for which the local density of states has a Lorentzian-like shape $[47,48]$. Thus, the scattering phase shift can be computed analytically and reads

$$
\delta_{i}^{\sigma}(\varepsilon)=\frac{\pi}{2}+\operatorname{atan}\left(\frac{\varepsilon-\varepsilon_{i}^{\sigma}}{\Gamma_{\sigma}}\right),
$$

where $\varepsilon_{i}^{\sigma}$ is the resonance position for the spin channel $\sigma$ and $\Gamma_{\sigma}$ is the resonance width at half maximum. The broadening of the impurity states is induced by hybridization with the Rashba electron gas and with other substrate electronic states not explicitly being considered. Furthermore, for the $3 d$ transition-metal impurities of interest, the majority spin is fully occupied [i.e., $\delta_{i}^{\uparrow}(\varepsilon)=\pi$ ] and does not contribute to the bound current density (see Sec. III).

The net orbital magnetization can be obtained by integrating the orbital magnetization density computed with Eq. (14) over the simulation box. Alternatively, we can provide an approximate connection between $M_{l, z}$ and the transition matrices [and therefore with $\delta_{i}^{\sigma}(\varepsilon)$ ] using the classical formula

$$
\vec{M}_{l}=\frac{1}{2} \int_{S} d \vec{r} \vec{r} \times \vec{j}(\vec{r})
$$

Starting from Eq. (11) and using the asymptotic forms of $G_{\mathrm{D}}(r, \varepsilon)$ and $G_{\mathrm{ND}}(r, \varepsilon)$ for $r \rightarrow \infty$ and then performing the spatial integral in the previous equation, we find the following approximate expression:

$$
M_{l, z}^{\mathrm{app}} \propto \operatorname{Re} \int_{0}^{\varepsilon_{\mathrm{F}}} d \varepsilon \frac{\Delta t_{i}(\varepsilon)}{\sqrt{\varepsilon}} .
$$

In Fig. 3, we show $M_{l, z}$ and $M_{l, z}^{\text {app }}$ as a function of the resonance position of the minority-spin channel $\varepsilon_{\downarrow} . \Delta t_{i}(\varepsilon)$ is computed using the energy-dependent scattering phase shift given in Eq. (18). Both quantities follow the same trend and display a steplike feature, showing a dependence on the valence of the impurity. Furthermore, since we assumed that the majority impurity resonance is fully occupied, in the limit $\varepsilon_{i}^{\downarrow} \rightarrow-\infty$, both resonances become fully occupied, and the net orbital magnetization vanishes. Moreover, when $\varepsilon_{i}^{\downarrow} \rightarrow$ $+\infty, M_{l, z}$ vanishes as well since the occupied Rashba states do not hybridize with the impurity states. This shows that the nature of the impurity has a deep impact on the induced orbital magnetization, and the valence of the impurity can be employed to tune its magnitude.

\section{ORBITAL MAGNETIZATION OF A TRIMER ON A RASHBA ELECTRON GAS}

After investigating the emerging orbital magnetization induced by a single magnetic impurity, we consider now a more complex nanostructure composed of three $\mathrm{Fe}$ atoms forming an equilateral triangle centered at the origin. The distance between the $\mathrm{Fe}$ impurities is $d=10.42 \AA$, corresponding to the seventh-nearest-neighbor distance on $\mathrm{Au}(111)$ (longdistance regime where the $s$-wave approximation is valid). For this separation the impurity $\mathrm{Fe}$ magnetic moments are

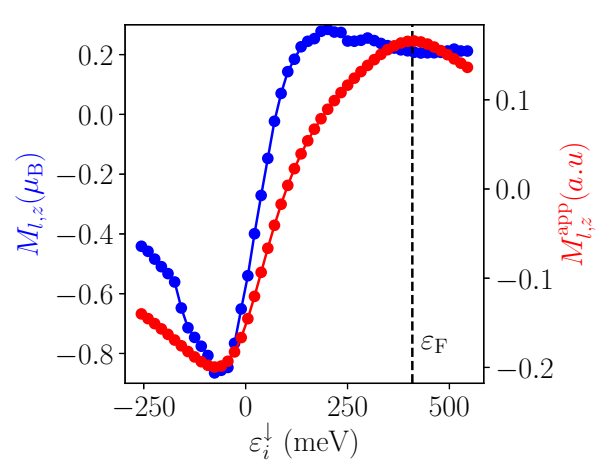

FIG. 3. Evolution of $M_{l, z}$ (blue curve) and of $M_{l, z}^{\text {app }}$ (red curve) as a function of the impurity resonance position $\varepsilon_{\downarrow}^{i}$ (minority-spin channel). The curves have a similar behavior and display a bandfilling effect. The broadening of the minority-spin channel is set to $\Gamma_{\downarrow}=115 \mathrm{meV}$, and the Fermi energy $\varepsilon_{\mathrm{F}}=410 \mathrm{meV}$. 
(a)

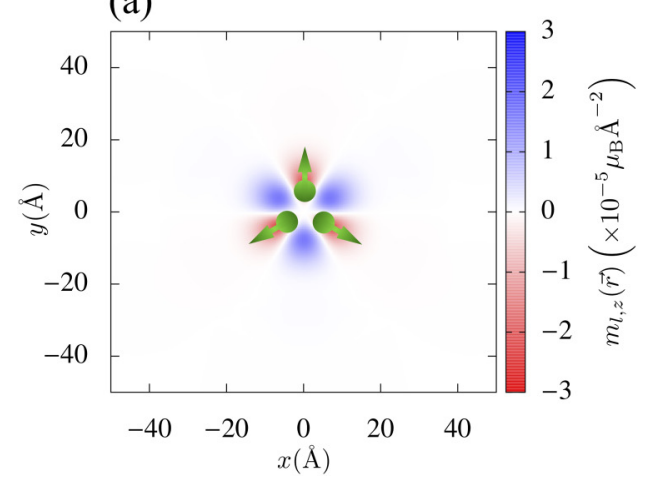

(b)

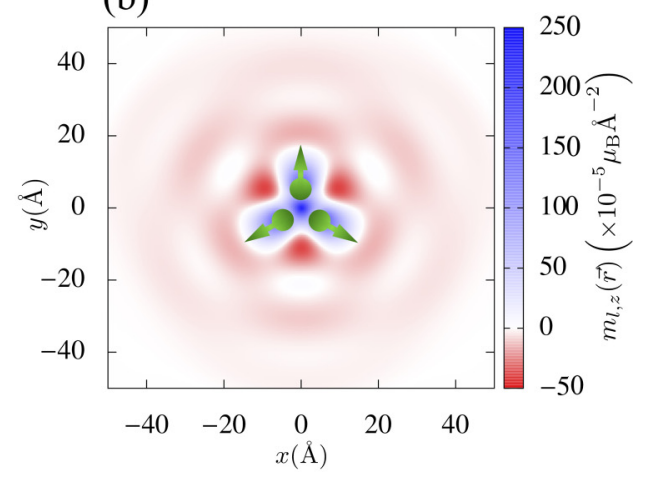

FIG. 4. Induced orbital magnetization map for an Fe trimer deposited on a Rashba electron gas with an equilateral-triangle geometry. We used the same model parameters as in Sec. III. The Fe impurities are represented by a green sphere located at the origin, while their magnetic moment is represented by a green arrow (the Fe impurities are considered in the $s$-wave approximation [15]). The magnetic trimer has an opening angle of $\theta=60^{\circ}$, and the azimuthal angles are $\phi_{i}=\left\{330^{\circ}, 90^{\circ}, 210^{\circ}\right\}$. (a) In the absence of SO interaction, the orbital magnetization remains finite; it follows $C_{3 v}$ symmetry and is rather small. (b) In the presence of SO interaction, the orbital magnetization is two orders of magnitude higher in comparison with the previous case and displays a constructive interference at the center of mass of the equilateral triangle.

coupled antiferromagnetically [40], leading to a noncollinear magnetic state. The ground state without the presence of spin-orbit interaction is a Néel state with an angle between the impurity spins of $120^{\circ}$. We first begin by omitting the contribution of the SO interaction and assume that the moments are noncoplanar with an opening polar angle of $\theta=60^{\circ}$ and an azimuthal angle $\phi_{i}=\left\{330^{\circ}, 90^{\circ}, 210^{\circ}\right\}$. The resulting orbital magnetization is shown in Fig. 4(a). Even though the SO interaction is absent, $m_{l, z}(\vec{r})$ is finite and follows the $C_{3 v}$ symmetry of the system [31]. In this case, the current density and, consequently, the induced angular momentum have their origin in the noncollinearity of the moments and can be traced to the scalar three-spin chirality $\vec{S}_{i} \cdot\left(\vec{S}_{j} \times \vec{S}_{k}\right)$ and its higher-order generalizations (see Appendix B). For that reason, we refer to this contribution as chiral orbital magnetization. Like in the single-atom case, $m_{l, z}(\vec{r})$ oscillates with two wavelengths $\left(\lambda_{\mathrm{F}}\right.$ and $\left.\lambda_{\mathrm{R}}\right)$. The results obtained in Fig. 4(a) also reveal that the induced net chiral orbital magnetization vanishes by symmetry in the simulation box. The net induced spin magnetization is, however, finite, $M_{s, z}=-0.16 \mu_{\mathrm{B}}$.

Including the contribution of the SO interaction, the obtained orbital magnetization is shown in Fig. 4(b). Like in the SO-interaction-free case, $m_{l, z}(\vec{r})$ obeys $C_{3 v}$ symmetry and has a $1 / r$ decay, but now with values two orders of magnitude larger than the chiral contribution. The oscillation in $m_{l, z}(\vec{r})$ is more pronounced, and the constructive interference at the center of mass of the triangle gives rise to large values of $m_{l, z}(\vec{r})$. The net induced spin and orbital magnetizations are $M_{s, z}=$ $-0.49 \mu_{\mathrm{B}}$ and $M_{l, z}=-0.35 \mu_{\mathrm{B}}$, respectively. In the presence of SO interaction, the connection between $m_{l, z}(\vec{r})$ and the noncollinear spin texture is more complex. It was shown previously in Ref. [38] that for continuous spin textures, the presence of two noncollinear spin moments is enough to influence the orbital magnetization. In the limit of fastrotating spin textures, similar terms arise in $M_{l, z}$. However, as shown in the following, higher-order contributions besides the three-spin chirality can be of crucial importance and strongly influence its angular dependence.

To study the dependence of the total induced orbital magnetization $M_{l, z}$ (including both chiral and SO contributions) on the spin orientation of the impurities, we computed it for different opening angles $\theta$. The result is shown in Fig. 5, where we notice that $M_{l, z}$ can be rather large, reaching $-0.4 \mu_{\mathrm{B}}$ for $\theta=70^{\circ}$. Then by performing a Born expansion of the Green function and retaining only terms up to first order in SO interaction (similar to what we do in Appendix B), $M_{l, z}$ can be written as

$$
\begin{aligned}
M_{l, z}= & \beta_{1} \cos \theta+\beta_{2} \cos \frac{\gamma}{2} \\
& +\beta_{3} \sin ^{2} \theta \cos \theta+\beta_{4} \cos ^{2} \frac{\gamma}{2} \\
& +\beta_{5} \cos \gamma \sin ^{2} \theta \cos \theta,
\end{aligned}
$$

where $\gamma=\arccos \left(\vec{S}_{1} \cdot \vec{S}_{2}\right)$ is the angle between $\vec{S}_{1}$ and $\vec{S}_{2}$. As shown in Appendix B, in the absence of SO interaction $\beta_{1}=\beta_{2}=\beta_{4}=0$, and the functional form includes only the odd powers of the spin moments. We also show in Fig. 5 the different fits of the orbital magnetization obtained when truncating Eq. (21) at different orders and provide the values

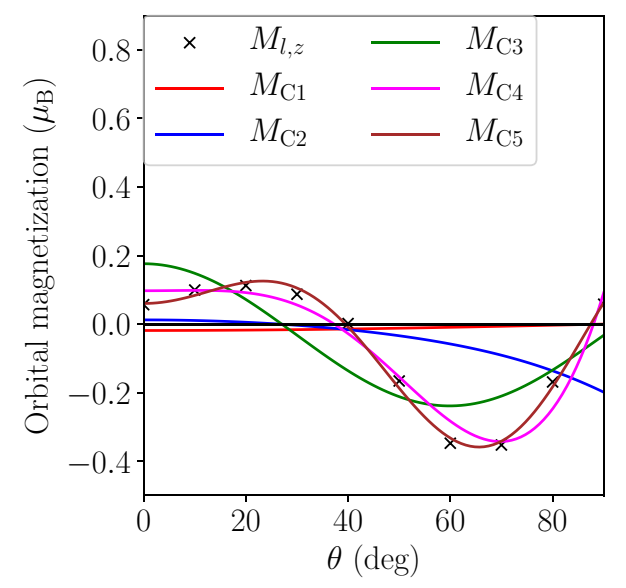

FIG. 5. Net orbital magnetization $M_{l, z}$ as a function of the opening polar angle $\theta$ of an $\mathrm{Fe}$ trimer in an equilateral-triangle geometry (see Fig. 4). The black crosses indicate the values of the net orbital magnetization computed using Eq. (15). The solid curves represent the fits of the net orbital magnetization up to different orders. 
TABLE I. Parameters used to fit the orbital magnetization up to the fifth power of spin impurity moment. $M_{\mathrm{C} i}$ stands for the fit of the orbital magnetization up to the $i$ th order. $C$ stands for chirality. The fits are displayed in Fig. 5.

\begin{tabular}{lrrrrr}
\hline \hline Parameter & \multicolumn{1}{c}{$\beta_{1}$} & \multicolumn{1}{c}{$\beta_{2}$} & $\beta_{3}$ & $\beta_{4}$ & $\beta_{5}$ \\
\hline$M_{\mathrm{C} 1}$ & -0.019 & & & & \\
$M_{\mathrm{C} 2}$ & 0.409 & -0.397 & & & \\
$M_{\mathrm{C} 3}$ & 0.242 & -0.065 & -0.843 & & \\
$M_{\mathrm{C} 4}$ & -7.653 & -7.385 & 4.817 & 15.136 & \\
$M_{\mathrm{C} 5}$ & 1.854 & 2.042 & -2.244 & -3.836 & 1.932 \\
\hline \hline
\end{tabular}

of the coefficients $\beta_{i}$ in Table I. This reveals the importance of higher-order contributions to capture the right angular dependence of $M_{l, z}$ in the entire range of angles taken into account. The low-order expansions would be able to reproduce the correct behavior only in a small angular window.

\section{DISCUSSION AND CONCLUSION}

In this paper, we used a model approach relying on the Rashba Hamiltonian to understand the emergence of an induced orbital magnetization when magnetic impurities are incorporated into a Rashba electron gas. The magnetic impurities were described using scattering phase shifts that either were taken to be constant or were obtained with a Lorentzian-like shape of the impurity density of states. We computed the dissipationless bound currents present in the system, which consist of paramagnetic and diamagneticlike contributions, devising a method applicable to any ensemble of impurities with an arbitrary magnetic configuration. Afterwards, we showed analytically that, in the presence of a single magnetic impurity with its moment parallel to the $z$ axis, a finite orbital magnetization arises when time- and space-inversion symmetries are simultaneously broken. The net orbital magnetization was found to be of the order of magnitude of its spin counterpart. However, it vanishes by symmetry when the impurity spin moment lies in the surface plane.

The dependence of the net orbital magnetization on the nature of the impurity was also addressed. Its magnitude and sign strongly depend on the impurity type (namely, its valence and the location of the impurity resonances with respect to the Fermi energy of the electron gas). Moreover, we considered a more complex magnetic structure consisting of a magnetic trimer in an equilateral geometry. In the absence of SO interaction and when the spin texture displays a nonvanishing scalar spin chirality, a chiral orbital magnetization is observed [31]. This result was also recovered analytically. When the SO interaction is turned on, the dependence of the net orbital magnetization on the spin texture is more complex, and higher-order powers of the spin chirality can be of crucial importance. In this case, the orbital magnetization density was two orders of magnitude higher than in the case where the SO interaction is not present.

Finally, we foresee the possibility of measuring the total (spin/orbital) induced magnetization at the surface utilizing spin-polarized STM [49]. Distinguishing, however, the spin from the orbital magnetization is not trivial. One has to con- sider the asymptotic behavior and the specific decay of both types of magnetization. Since the magnetization density produces stray fields, measurements exploiting nitrogen-vacancy centers might enable their detection [50]. Moreover, we believe that XMCD is a possible technique to track higher-order spin chiralities, which play a major role in the determination of induced orbital magnetization by altering the topological properties of the orbital magnetization induced by complex magnetic structures such as magnetic skyrmions [31].

\section{ACKNOWLEDGMENTS}

This work was supported by the European Research Council (ERC) under the European Union's Horizon 2020 research and innovation program (ERC-consolidator Grant No. 681405 DYNASORE).

\section{APPENDIX A: SINGLE-MAGNETIC-ADATOM GROUND-STATE CHARGE CURRENT}

In this appendix, we derive the ground-state charge current induced by magnetic impurities with a spin moment perpendicular to the plane containing the Rashba electron gas (i.e., along the $z$ axis). The current operator given in Eq. (6) contains a gradient acting on the Green's function. Since the cylindrical symmetry of the Rashba electron gas is preserved when the moment points out of the plane, we write the gradient in cylindrical coordinates as

$$
\vec{\nabla}_{\vec{r}}=\vec{e}_{r} \frac{\partial}{\partial r}+\frac{1}{r} \vec{e}_{\theta} \frac{\partial}{\partial \theta}
$$

Here, $\vec{e}_{r}$ and $\vec{e}_{\theta}$ are the radial and azimuthal unit vectors, respectively. Assuming an impurity located at position $\vec{R}_{i}$, we define the gradient accordingly as $\vec{\nabla}_{\vec{r}_{i}}$. Furthermore, the Rashba Green's function is a matrix in spin space, given by [43]

$$
\boldsymbol{G}^{\mathrm{R}}\left(\vec{R}, \varepsilon+i 0^{+}\right)=\left(\begin{array}{cc}
G_{\mathrm{D}}(R, \varepsilon) & -G_{\mathrm{ND}}(R, \varepsilon) e^{-i \beta} \\
G_{\mathrm{ND}}(R, \varepsilon) e^{i \beta} & G_{\mathrm{D}}(R, \varepsilon)
\end{array}\right)
$$

where $\vec{R}=\vec{r}-\vec{r}^{\prime}$ and $\beta$ is the angle between $\vec{R}$ and the $x$ axis. $G_{\mathrm{D}}(R, \varepsilon)$ and $G_{\mathrm{ND}}(R, \varepsilon)$ are given by linear combinations of Hankel functions of zeroth and first order, respectively,

$$
\begin{aligned}
G_{\mathrm{D}}\left(R, \varepsilon+i 0^{+}\right)= & -\frac{i m^{*}}{2 \hbar^{2}\left(k_{+}+k_{-}\right)}\left[k_{+} H_{0}\left(k_{+} R+i 0^{+}\right)\right. \\
& \left.+k_{-} H_{0}\left(k_{-} R+i 0^{+}\right)\right], \\
G_{\mathrm{ND}}\left(R, \varepsilon+i 0^{+}\right)= & -\frac{i m^{*}}{2 \hbar^{2}\left(k_{+}+k_{-}\right)}\left[k_{+} H_{1}\left(k_{+} R+i 0^{+}\right)\right. \\
& \left.-k_{-} H_{1}\left(k_{-} R+i 0^{+}\right)\right] .
\end{aligned}
$$

The wave vectors $k_{+}$and $k_{-}$are given by $k_{+}=k_{\mathrm{R}}+$ $\sqrt{k_{\mathrm{R}}^{2}+\frac{2 m^{*} \varepsilon}{\hbar^{2}}}$ and $k_{-}=-k_{\mathrm{R}}+\sqrt{k_{\mathrm{R}}^{2}+\frac{2 m^{*} \varepsilon}{\hbar^{2}}}$, with $k_{\mathrm{R}}=\frac{m^{*} \alpha_{\mathrm{R}}}{\hbar^{2}}$. 
The gradient of the Rashba Green's function is given by

$$
\vec{\nabla}_{\vec{r}_{i}} \boldsymbol{G}^{\mathrm{R}}\left(\vec{r}_{i}, \varepsilon+i 0^{+}\right)=\left(\begin{array}{cc}
\vec{e}_{r_{i}} \frac{\partial G_{\mathrm{D}}}{\partial r_{i}} & e^{-i \theta_{i}}\left[\begin{array}{c}
\left.-\vec{e}_{r_{i}} \frac{\partial G_{\mathrm{ND}}}{\partial r_{i}}+i \vec{e}_{\theta_{i}} \frac{G_{\mathrm{ND}}}{r_{i}}\right] \\
e^{i \theta_{i}}\left[\vec{e}_{r_{i}} \frac{\partial G_{\mathrm{ND}}}{\partial r_{i}}+i \vec{e}_{\theta_{i}} \frac{G_{\mathrm{ND}}}{r_{i}}\right]
\end{array}\right. \\
\vec{e}_{r_{i}} \frac{\partial G_{\mathrm{D}}}{\partial r_{i}}
\end{array}\right),
$$

with $\vec{r}_{i}=\vec{r}-\vec{R}_{i}$ and $\vec{R}_{i}$ being the position of the impurity $i$.

From Eq. (A5) $\frac{\partial G_{\mathrm{D}}}{\partial r_{i}}$ and $\frac{\partial G_{\mathrm{ND}}}{\partial r_{i}}$ are needed. This involves first-order derivatives of Hankel functions, which can be computed using recursion:

$$
\frac{d H_{n}(x)}{d x}=\left[\frac{n H_{n}(x)}{x}-H_{n+1}(x)\right]
$$

For the Rashba Green's function one needs the derivatives of $H_{0}(x)$ and $H_{1}(x)$ :

$$
\begin{aligned}
& \frac{d H_{0}(x)}{d x}=-H_{1}(x), \\
& \frac{d H_{1}(x)}{d x}=\left[\frac{H_{1}(x)}{x}-H_{2}(x)\right] .
\end{aligned}
$$

After computing $\vec{\nabla}_{\vec{r}_{i}} \boldsymbol{G}^{\mathrm{R}}\left(\vec{r}_{i}, \varepsilon+i 0^{+}\right)$, one can easily access $\vec{\nabla}_{\vec{r}_{i}} \boldsymbol{G}\left(\vec{r}_{i}, \varepsilon+i 0^{+}\right)$via Eq. (8), which is employed to compute the expectation value of $\vec{j}$ given in Eq. (6) via

$$
\vec{j}(\vec{r})=\int_{-\infty}^{\varepsilon_{\mathrm{F}}} d \varepsilon \operatorname{Tr} \vec{j} \boldsymbol{G}(\vec{r}, \varepsilon),
$$

where the trace is taken over the spin degree of freedom.

\section{APPENDIX B: PARAMAGNETIC CHARGE CURRENT WITHOUT SO INTERACTION}

Here, we derive the connection between the chiral orbital magnetization and the scalar chirality (and the spin texture in general) up to fifth order. We consider that the spin-orbit interaction is zero (i.e., $\alpha_{R}=0$ ); thus, the Rashba Green's function becomes spin diagonal. Then we perform a Born expansion of Eq. (9) as

$$
\begin{aligned}
\boldsymbol{G}\left(\vec{r}, \vec{r}^{\prime}, \varepsilon\right)= & \boldsymbol{G}^{\mathrm{R}}\left(\vec{r}, \vec{r}^{\prime}, \varepsilon\right)+\boldsymbol{G}^{(1)}\left(\vec{r}, \vec{r}^{\prime}, \varepsilon\right) \\
& +\boldsymbol{G}^{(2)}\left(\vec{r}, \vec{r}^{\prime}, \varepsilon\right)+\boldsymbol{G}^{(3)}\left(\vec{r}, \vec{r}^{\prime}, \varepsilon\right) \\
& +\boldsymbol{G}^{(4)}\left(\vec{r}, \vec{r}^{\prime}, \varepsilon\right)+\boldsymbol{G}^{(5)}\left(\vec{r}, \vec{r}^{\prime}, \varepsilon\right)+\cdots
\end{aligned}
$$

The different elements of the expansion $G^{(i)}\left(\vec{r}, \vec{r}^{\prime}, \varepsilon\right)$ are

$$
\begin{aligned}
\boldsymbol{G}^{(1)}\left(\vec{r}, \vec{r}^{\prime}, \varepsilon\right)= & \sum_{i} \boldsymbol{G}^{\mathrm{R}}\left(\vec{r}, \vec{r}_{i}, \varepsilon\right) \boldsymbol{t}_{i}(\varepsilon) \boldsymbol{G}^{\mathrm{R}}\left(\vec{r}_{i}, \vec{r}^{\prime}, \varepsilon\right), \\
\boldsymbol{G}^{(2)}\left(\vec{r}, \vec{r}^{\prime}, \varepsilon\right)= & \sum_{i j} \boldsymbol{G}^{\mathrm{R}}\left(\vec{r}, \vec{r}_{i}, \varepsilon\right) \boldsymbol{t}_{i}(\varepsilon) \boldsymbol{G}^{\mathrm{R}}\left(\vec{r}_{i}, \vec{r}_{j}, \varepsilon\right) \\
& \times \boldsymbol{t}_{j}(\varepsilon) \boldsymbol{G}^{\mathrm{R}}\left(\vec{r}_{j}, \vec{r}^{\prime}, \varepsilon\right), \\
\boldsymbol{G}^{(3)}\left(\vec{r}, \vec{r}^{\prime}, \varepsilon\right)= & \sum_{i j k} \boldsymbol{G}^{\mathrm{R}}\left(\vec{r}, \vec{r}_{i}, \varepsilon\right) \boldsymbol{t}_{i}(\varepsilon) \boldsymbol{G}^{\mathrm{R}}\left(\vec{r}_{i}, \vec{r}_{j}, \varepsilon\right) \boldsymbol{t}_{j}(\varepsilon) \\
& \times \boldsymbol{G}^{\mathrm{R}}\left(\vec{r}_{j}, \vec{r}_{k}, \varepsilon\right) \boldsymbol{t}_{k}(\varepsilon) \boldsymbol{G}^{\mathrm{R}}\left(\vec{r}_{k}, \vec{r}^{\prime}, \varepsilon\right),
\end{aligned}
$$

$$
\begin{aligned}
\boldsymbol{G}^{(4)}\left(\vec{r}, \vec{r}^{\prime}, \varepsilon\right)= & \sum_{i j k m} \boldsymbol{G}^{\mathrm{R}}\left(\vec{r}, \vec{r}_{i}, \varepsilon\right) \boldsymbol{t}_{i}(\varepsilon) \boldsymbol{G}^{\mathrm{R}}\left(\vec{r}_{i}, \vec{r}_{j}, \varepsilon\right) \boldsymbol{t}_{j}(\varepsilon) \\
& \times \boldsymbol{G}^{\mathrm{R}}\left(\vec{r}_{j}, \vec{r}_{k}, \varepsilon\right) \boldsymbol{t}_{k}(\varepsilon) \boldsymbol{G}^{\mathrm{R}}\left(\vec{r}_{k}, \vec{r}_{m}, \varepsilon\right) \\
& \times \boldsymbol{t}_{m}(\varepsilon) \boldsymbol{G}^{\mathrm{R}}\left(\vec{r}_{m}, \vec{r}^{\prime}, \varepsilon\right),
\end{aligned}
$$

$$
\begin{aligned}
\boldsymbol{G}^{(5)}\left(\vec{r}, \vec{r}^{\prime}, \varepsilon\right)= & \sum_{i j k} \boldsymbol{G}^{\mathrm{R}}\left(\vec{r}, \vec{r}_{i}, \varepsilon\right) \boldsymbol{t}_{i}(\varepsilon) \boldsymbol{G}^{\mathrm{R}}\left(\vec{r}_{i}, \vec{r}_{j}, \varepsilon\right) \boldsymbol{t}_{j}(\varepsilon) \\
& \times \boldsymbol{G}^{\mathrm{R}}\left(\vec{r}_{j}, \vec{r}_{k}, \varepsilon\right) \boldsymbol{t}_{k}(\varepsilon) \boldsymbol{G}^{\mathrm{R}}\left(\vec{r}_{k}, \vec{r}^{\prime}, \varepsilon\right) \boldsymbol{t}_{m}(\varepsilon) \\
& \times \boldsymbol{G}^{\mathrm{R}}\left(\vec{r}_{m}, \vec{r}^{\prime}, \varepsilon\right) \boldsymbol{t}_{l}(\varepsilon) \boldsymbol{G}^{\mathrm{R}}\left(\vec{r}_{l}, \vec{r}^{\prime}, \varepsilon\right) .
\end{aligned}
$$

In the absence of spin-orbit interaction, the current operator given in Eq. (6) contains only the paramagnetic part and reduces to $\frac{e \hbar}{2 m^{*} i} \lim _{\vec{r}^{\prime} \rightarrow \vec{r}}\left(\vec{\nabla}_{\vec{r}}-\vec{\nabla}_{\vec{r}^{\prime}}\right)$. Therefore, the current density is given by

$$
\vec{j}(\vec{r})=\vec{j}^{(3)}(\vec{r})+\vec{j}^{(5)}(\vec{r}),
$$

where due to the cyclic properties of the trace, only the odd powers of the expansion contribute to the current (the firstorder vanishes by symmetry). Furthermore, since $\boldsymbol{G}^{\mathrm{R}}\left(\vec{r}, \vec{r}^{\prime}, \varepsilon\right)$ is spin diagonal, it can be taken out of the trace, and $\vec{j}^{(3)}(\vec{r})$ is simply given by

$$
\begin{aligned}
\vec{j}^{(3)}(\vec{r}) \propto & \sum_{i j k} \vec{\nabla}_{\vec{r}} \boldsymbol{G}^{\mathrm{R}}\left(\vec{r}, \vec{r}_{i}, \varepsilon\right) \boldsymbol{G}^{\mathrm{R}}\left(\vec{r}_{i}, \vec{r}_{j}, \varepsilon\right) \boldsymbol{G}^{\mathrm{R}}\left(\vec{r}_{j}, \vec{r}_{k}, \varepsilon\right) \\
& \times \boldsymbol{G}^{\mathrm{R}}\left(\overrightarrow{r_{k}}, \vec{r}, \varepsilon\right) \operatorname{Tr}\left[\boldsymbol{t}_{i}(\varepsilon) \boldsymbol{t}_{j}(\varepsilon) \boldsymbol{t}_{k}(\varepsilon)\right. \\
& \left.-\boldsymbol{t}_{k}(\varepsilon) \boldsymbol{t}_{j}(\varepsilon) \boldsymbol{t}_{i}(\varepsilon)\right],
\end{aligned}
$$

while the fifth-order contribution reads

$$
\begin{aligned}
\vec{j}^{(5)}(\vec{r}) \propto & \sum_{i j k m l} \vec{\nabla}_{\vec{r}} \boldsymbol{G}^{\mathrm{R}}\left(\vec{r}, \vec{r}_{i}, \varepsilon\right) \boldsymbol{G}^{\mathrm{R}}\left(\vec{r}_{i}, \vec{r}_{j}, \varepsilon\right) \boldsymbol{G}^{\mathrm{R}}\left(\vec{r}_{j}, \vec{r}_{k}, \varepsilon\right) \\
& \times \boldsymbol{G}^{\mathrm{R}}\left(\vec{r}_{k}, \vec{r}_{m}, \varepsilon\right) \boldsymbol{G}^{\mathrm{R}}\left(\vec{r}_{m}, \vec{r}_{l}, \varepsilon\right) \boldsymbol{G}^{\mathrm{R}}\left(\vec{r}_{l}, \vec{r}, \varepsilon\right) \\
& \times \operatorname{Tr}\left[\boldsymbol{t}_{i}(\varepsilon) \boldsymbol{t}_{j}(\varepsilon) \boldsymbol{t}_{k}(\varepsilon) \boldsymbol{t}_{m}(\varepsilon) \boldsymbol{t}_{l}(\varepsilon)\right. \\
& \left.-\boldsymbol{t}_{l}(\varepsilon) \boldsymbol{t}_{m}(\varepsilon) \boldsymbol{t}_{k}(\varepsilon) \boldsymbol{t}_{j}(\varepsilon) \boldsymbol{t}_{i}(\varepsilon)\right] .
\end{aligned}
$$

Further simplifications can be made to the expressions given in Eqs. (B8) and (B9), considering that

$$
\boldsymbol{t}_{i}=\frac{t_{i}^{\uparrow}+t_{i}^{\downarrow}}{2}+\frac{t_{i}^{\uparrow}-t_{i}^{\downarrow}}{2} \overrightarrow{\boldsymbol{\sigma}} \cdot \vec{S}_{i}
$$

and using the properties of the Pauli matrices, $\vec{j}^{(3)}(\vec{r})$ simplifies to

$$
\vec{j}^{(3)}(\vec{r})=\sum_{i j k} \vec{f}_{3}(\vec{r}) \vec{S}_{i} \cdot\left(\vec{S}_{j} \times \vec{S}_{k}\right)
$$


where $\vec{f}_{3}(\vec{r})$ is given by

$$
\begin{aligned}
\vec{f}_{3}(\vec{r})= & -\frac{2 e \hbar}{\pi m^{*}} \operatorname{Im} \vec{\nabla}_{\vec{r}} \boldsymbol{G}^{\mathrm{R}}\left(\vec{r}, \vec{r}_{i}, \varepsilon\right) \boldsymbol{G}^{\mathrm{R}}\left(\vec{r}_{i}, \vec{r}_{j}, \varepsilon\right) \\
& \times \boldsymbol{G}^{\mathrm{R}}\left(\vec{r}_{j}, \vec{r}_{k}, \varepsilon\right) \boldsymbol{G}^{\mathrm{R}}\left(\vec{r}_{k}, \vec{r}, \varepsilon\right) .
\end{aligned}
$$

The fifth-order contribution can also be simplified to

$$
\vec{j}^{(5)}(\vec{r})=\sum_{i j k m l} \vec{f}_{5}(\vec{r})\left(\vec{S}_{i} \cdot \vec{S}_{j}\right)\left[\vec{S}_{k} \cdot\left(\vec{S}_{m} \times \vec{S}_{l}\right)\right]
$$

where $\vec{f}_{5}(\vec{r})$ reads

$$
\begin{aligned}
\vec{f}_{5}(\vec{r})= & -\frac{4 e \hbar}{\pi m^{*}} \operatorname{Im} \vec{\nabla}_{\vec{r}} \boldsymbol{G}^{\mathrm{R}}\left(\vec{r}, \vec{r}_{i}, \varepsilon\right) \boldsymbol{G}^{\mathrm{R}}\left(\vec{r}_{i}, \vec{r}_{j}, \varepsilon\right) \boldsymbol{G}^{\mathrm{R}}\left(\vec{r}_{j}, \vec{r}_{k}, \varepsilon\right) \\
& \times \boldsymbol{G}^{\mathrm{R}}\left(\vec{r}_{k}, \vec{r}_{m}, \varepsilon\right) \boldsymbol{G}^{\mathrm{R}}\left(\vec{r}_{m}, \vec{r}_{l}, \varepsilon\right) \boldsymbol{G}^{\mathrm{R}}\left(\vec{r}_{l}, \vec{r}, \varepsilon\right) . \quad(\mathrm{B} 14)
\end{aligned}
$$

The previous equations show that in the absence of the spin-orbit interaction the induced bound currents (i.e., orbital magnetization) can be expanded as a function of the odd powers of the spin chirality.
[1] F. Hellman, A. Hoffmann, Y. Tserkovnyak, G. S. D. Beach, E. E. Fullerton, C. Leighton, A. H. MacDonald, D. C. Ralph, D. A. Arena, H. A. Dürr, P. Fischer, J. Grollier, J. P. Heremans, T. Jungwirth, A. V. Kimel, B. Koopmans, I. N. Krivorotov, S. J. May, A. K. Petford-Long, J. M. Rondinelli, N. Samarth, I. K. Schuller, A. N. Slavin, M. D. Stiles, O. Tchernyshyov, A. Thiaville, and B. L. Zink, Interface-induced phenomena in magnetism, Rev. Mod. Phys. 89, 025006 (2017).

[2] J. Sinova, S. O. Valenzuela, J. Wunderlich, C. H. Back, and T. Jungwirth, Spin Hall effects, Rev. Mod. Phys. 87, 1213 (2015).

[3] A. Manchon, H. C. Koo, J. Nitta, S. M. Frolov, and R. A. Duine, New perspectives for Rashba spin-orbit coupling, Nat. Mater. 14, 871 (2015).

[4] E. I. Rashba, Properties of semiconductors with a loop of extrema, I. Cyclotron and combined resonances in a perpendicular field, Sov. Phys. Solid State 2, 1109 (1960).

[5] E. I. Rashba and Y. A. Bychkov, Oscillatory effects and the magnetic susceptibility of carriers in inversion layers, J. Phys. C 17, 6039 (1984).

[6] S. LaShell, B. A. McDougall, and E. Jensen, Spin Splitting of an $\mathrm{Au}(111)$ Surface State Band Observed with Angle Resolved Photoelectron Spectroscopy, Phys. Rev. Lett. 77, 3419 (1996).

[7] F. Reinert, G. Nicolay, S. Schmidt, D. Ehm, and S. Hüfner, Direct measurements of the $L$-gap surface states on the (111) face of noble metals by photoelectron spectroscopy, Phys. Rev. B 63, 115415 (2001).

[8] G. Nicolay, F. Reinert, S. Hüfner, and P. Blaha, Spin-orbit splitting of the L-gap surface state on $\mathrm{Au}(111)$ and $\mathrm{Ag}(111)$, Phys. Rev. B 65, 033407 (2001).

[9] G. Bihlmayer, O. Rader, and R. Winkler, Focus on the Rashba effect, New J. Phys. 17, 050202 (2015).

[10] I. Gierz, T. Suzuki, E. Frantzeskakis, S. Pons, S. Ostanin, A. Ernst, J. Henk, M. Grioni, K. Kern, and C. R. Ast, Silicon Surface with Giant Spin Splitting, Phys. Rev. Lett. 103, 046803 (2009).

[11] M. Liebmann, C. Rinaldi, D. Di Sante, J. Kellner, C. Pauly, R. N. Wang, J. E. Boschker, A. Giussani, S. Bertoli, M. Cantoni, L. Baldrati, M. Asa, I. Vobornik, G. Panaccione, D. Marchenko, J. Sánchez-Barriga, O. Rader, R. Calarco, S. Picozzi, R. Bertacco, and M. Morgenstern, Giant Rashba-type spin splitting in ferroelectric GeTe(111), Adv. Mater. 28, 560 (2015).

[12] H. J. Elmers, R. Wallauer, M. Liebmann, J. Kellner, M. Morgenstern, R. N. Wang, J. E. Boschker, R. Calarco, J. SánchezBarriga, O. Rader, D. Kutnyakhov, S. V. Chernov, K. Medjanik, C. Tusche, M. Ellguth, H. Volfova, St. Borek, J. Braun, J. Minár, H. Ebert, and G. Schönhense, Spin mapping of surface and bulk Rashba states in ferroelectric $\alpha$-GeTe(111) films, Phys. Rev. B 94, 201403 (2016).
[13] J. Friedel, Metallic alloys, Nuovo Cimento 7, 287 (1958).

[14] M. F. Crommie, C. P. Lutz, and D. M. Eigler, Confinement of electrons to quantum corrals on a metal surface, Science 262, 218 (1993).

[15] G. A. Fiete and E. J. Heller, Colloquium: Theory of quantum corrals and quantum mirages, Rev. Mod. Phys. 75, 933 (2003).

[16] L. Petersen and P. Hedegård, A simple tight-binding model of spin-orbit splitting of sp-derived surface states, Surf. Sci. 459, 49 (2000).

[17] J. D. Walls and E. J. Heller, Spin-orbit coupling induced interference in quantum corrals, Nano Lett. 7, 3377 (2007).

[18] S. Lounis, A. Bringer, and S. Blügel, Magnetic Adatom Induced Skyrmion-Like Spin Texture in Surface Electron Waves, Phys. Rev. Lett. 108, 207202 (2012).

[19] T. Kikuchi, T. Koretsune, R. Arita, and G. Tatara, Dzyaloshinskii-Moriya Interaction as a Consequence of a Doppler Shift due to Spin-Orbit-Induced Intrinsic Spin Current, Phys. Rev. Lett. 116, 247201 (2016).

[20] T. Koretsune, T. Kikuchi, and R. Arita, First-principles evaluation of the Dzyaloshinskii-Moriya interaction, J. Phys. Soc. Jpn. 87, 041011 (2018).

[21] F. Freimuth, S. Blügel, and Y. Mokrousov, Relation of the Dzyaloshinskii-Moriya interaction to spin currents and to the spin-orbit field, Phys. Rev. B 96, 054403 (2017).

[22] A. N. Bogdanov and D. A. Yablonskii, Thermodynamically stable "vortices" in magnetically ordered crystals. The mixed state of magnets, Zh. Eksp. Teor. Fiz. 95, 182 (1989).

[23] N. Nagaosa and Y. Tokura, Topological properties and dynamics of magnetic skyrmions, Nat. Nanotechnol. 8, 899 (2013).

[24] A. Fert, N. Reyren, and V. Cros, Magnetic skyrmions: Advances in physics and potential applications, Nat. Rev. Mater. 2, 17031 (2017).

[25] A. Fert, V. Cros, and J. Sampaio, Skyrmions on the track, Nat. Nanotechnol. 8, 152 (2013).

[26] E. I. Rashba and I. I. Boiko, The properties of semiconductors with extremum loops. 3. Behavior in a magnetic field parallel to the plane of the loop, Sov. Phys. Solid State 3, 922 (1961).

[27] G. A. H. Schober, H. Murakawa, M. S. Bahramy, R. Arita, Y. Kaneko, Y. Tokura, and N. Nagaosa, Mechanisms of Enhanced Orbital Dia- and Paramagnetism: Application to the Rashba Semiconductor BiTeI, Phys. Rev. Lett. 108, 247208 (2012).

[28] S. S. Pershoguba, K. Björnson, A. M. Black-Schaffer, and A. V. Balatsky, Currents Induced by Magnetic Impurities in Superconductors with Spin-Orbit Coupling, Phys. Rev. Lett. 115, 116602 (2015).

[29] T. Thonhauser, Theory of orbital magnetization in solids, Int. J. Mod. Phys. B 25, 1429 (2011). 
[30] G. Tatara and N. Garcia, Quantum Toys for Quantum Computing: Persistent Currents Controlled by the Spin Josephson Effect, Phys. Rev. Lett. 91, 076806 (2003).

[31] M. dos Santos Dias, J. Bouaziz, M. Bouhassoune, S. Blügel, and S. Lounis, Chirality-driven orbital magnetic moments as a new probe for topological magnetic structures, Nat. Commun. 7, 13613 (2016).

[32] M. dos Santos Dias and S. Lounis, Insights into the orbital magnetism of noncollinear magnetic systems, Proc. SPIE 10357, 103572A (2017).

[33] D. Xiao, M. C. Chang, and Q. Niu, Berry phase effects on electronic properties, Rev. Mod. Phys. 82, 1959 (2010).

[34] K. Everschor-Sitte and M. Sitte, Real-space Berry phases: Skyrmion soccer (invited), J. Appl. Phys. 115, 172602 (2014).

[35] M. Hoffmann, J. Weischenberg, B. Dupé, F. Freimuth, P. Ferriani, Y. Mokrousov, and S. Heinze, Topological orbital magnetization and emergent Hall effect of an atomic-scale spin lattice at a surface, Phys. Rev. B 92, 020401 (2015).

[36] J. P. Hanke, F. Freimuth, A. K. Nandy, H. Zhang, S. Blügel, and Y. Mokrousov, Role of Berry phase theory for describing orbital magnetism: From magnetic heterostructures to topological orbital ferromagnets, Phys. Rev. B 94, 121114 (2016).

[37] J.-P. Hanke, F. Freimuth, S. Blügel, and Y. Mokrousov, Prototypical topological orbital ferromagnet g-FeMn, Sci. Rep. 7, 41078 (2017).

[38] F. R. Lux, F. Freimuth, S. Blügel, and Y. Mokrousov, Chiral and topological orbital magnetism of spin textures, arXiv: 1706.06068 .

[39] A. Dyrdał, V. K. Dugaev, and J. Barnaś, Spin-resolved orbital magnetization in Rashba two-dimensional electron gas, Phys. Rev. B 94, 205302 (2016).

[40] J. Bouaziz, M. dos Santos Dias, A. Ziane, M. Benakki, S. Blügel, and S. Lounis, Chiral magnetism of magnetic adatoms generated by Rashba electrons, New J. Phys. 19, 023010 (2017).

[41] N. Hatano, R. Shirasaki, and H. Nakamura, Non-Abelian gauge field theory of the spin-orbit interaction and a perfect spin filter, Phys. Rev. A 75, 032107 (2007).

[42] S.-H. Chen and C.-R. Chang, Non-Abelian spin-orbit gauge: Persistent spin helix and quantum square ring, Phys. Rev. B 77, 045324 (2008).

[43] J. D. Walls, J. Huang, R. M. Westervelt, and E. J. Heller, Multiple-scattering theory for two-dimensional electron gases in the presence of spin-orbit coupling, Phys. Rev. B 73, 035325 (2006).

[44] B. Berche and E. Medina, Classical Yang-Mills theory in condensed matter physics, Eur. J. Phys. 34, 161 (2013).

[45] S. S. Pershoguba, S. Nakosai, and A. V. Balatsky, Skyrmioninduced bound states in a superconductor, Phys. Rev. B 94, 064513 (2016).

[46] L. L. Hirst, The microscopic magnetization: Concept and application, Rev. Mod. Phys. 69, 607 (1997).

[47] S. Lounis, P. Mavropoulos, P. H. Dederichs, and S. Blügel, Surface-state scattering by adatoms on noble metals: Ab initio calculations using the Korringa-Kohn-Rostoker Green function method, Phys. Rev. B 73, 195421 (2006).

[48] V. S. Stepanyuk, W. Hergert, K. Wildberger, R. Zeller, and P. H. Dederichs, Magnetism of $3 d, 4 d$, and $5 d$ transition-metal impurities on $\operatorname{Pd}(001)$ and $\operatorname{Pt}(001)$ surfaces, Phys. Rev. B 53, 2121 (1996).

[49] F. Meier, S. Lounis, J. Wiebe, L. Zhou, S. Heers, P. Mavropoulos, P. H. Dederichs, S. Blügel, and R. Wiesendanger, Spin polarization of platinum (111) induced by the proximity to cobalt nanostripes, Phys. Rev. B 83, 075407 (2011).

[50] D. M. Toyli, D. J. Christle, A. Alkauskas, B. B. Buckley, C. G. Van de Walle, and D. D. Awschalom, Measurement and Control of Single Nitrogen-Vacancy Center Spins above 600 K, Phys. Rev. X 2, 031001 (2012). 